\title{
Can Semaglutide Bind Tighter to GLP-1R?
}

\author{
Wei Li *
}

July 18, 2020

\begin{abstract}
Semaglutide is a glucagon-like peptide 1 analog used for the treatment of patients with type 2 diabetes mellitus. With $94 \%$ sequence similarity to human GLP-1, semaglutide is a glucagon-like peptide-1 receptor (GLP-1R) agonist, which binds directly to GLP-1R, causing various beneficial downstream effects that reduce blood glucose. Practically, it is favourable for semaglutide to bind not just directly, but also tighter, to its receptor GLP-1R. Therefore, incorporating currently available experimental structural data of semaglutide-GLP-1R, this short article reports for the first time that biophysically, semaglutide is able to bind tighter to GLP-1R with just a simple Val27-Arg28 exchange in its peptide backbone.
\end{abstract}

*Institute of Special Environmental Medicine, Nantong University, No. 9, Seyuan Road, Nantong City, Jiangsu Province, People's Republic of China 


\section{Introduction}

Semaglutide is a glucagon-like peptide 1 (GLP-1) analog used for the treatment of patients with type 2 diabetes mellitus along with lifestyle changes [1, 2, 3, 4, 5, 6, 7]. Other members of this drug class include exenatide and liraglutide $[8,9,10,11,12$, 13, 14, 15, 16]. Semaglutide was developed by Novo Nordisk and approved by FDA for subcutaneous injection in December 2017, the tablet formulation was approved for oral administration in September 2019 [2, 11, 17, 18, 19, 20, 21, 22, 23, 24, 25, 26, 27]. Semaglutide offers a competitive advantage over other drugs used to treat diabetes, which may require several daily doses. Clinical trials have revealed that this drug reduces glycosylated hemoglobin levels and reduces body weight, proving to be effective for patients with type 2 diabetes [1, 3, 28, 10, 29, 30, 31, 32, 33].

Pharmacologically, semaglutide stimulates the synthesis of insulin by stimulating pancreatic islet cells and reducing glucagon secretion $[34,35]$. In hypercholesterolemia, semaglutide is believed to reduce the progression of atherosclerosis via decreased gut permeability and decreased inflammation [36, 11], while weight loss is believed to occur via the reduction of appetite and food cravings after semaglutide administration $[37,35]$.

\section{Motivation}

With 94\% sequence similarity to human GLP-1, semaglutide is a glucagon-like peptide1 receptor (GLP-1R) agonist $[11,17,18,38,1,34,39]$, which binds directly to GLP-1R, causing various beneficial downstream effects that reduce blood glucose $[40,41]$. As of July 18, 2020, there is already one experimental structure (determined by X-ray diffraction) of the semaglutide peptide backbone in complex with the extracellular 
domain of GLP-1R (PDB ID: 4ZGM [2]). Therefore, incorporating this structure (PDB ID: 4ZGM [2]), this short article aims to answer: biophysically, can semaglutide bind tighter to GLP-1R?

\section{Materials and Methods}

To begin with, a close inspection and structural analysis [42] of the binding interface of the complex structure (PDB ID: 4ZGM [2]) led to a B27Arg-B28Val mutant of semaglutide with potentially higher binding affinity to GLP-1R. Specifically, the amino acid sequence of GLP-1R is listed in italics in fasta format as below,

$>$ GLP-1R

RPQGATVSLWETVQKWREYRRQCQRSLTEDPPPATDLFCNRTFDEYAC WPDGEPGSFVNVSCPWYLPWASSVPQGHVYRFCTAEGLWLQKDNSSLPWR DLSECEESKRGERSSPEEQLLFLY

The amino acid sequence of native semaglutide peptide backbone is listed in italics in fasta format as below,

$>$ SemaglutideNative

HAEGTFTSDVSSYLEGQAAKEFIA WLVRGRG

The amino acid sequence of a B27Arg-B28Val mutant semaglutide peptide backbone is listed in italics in fasta format as below,

$>$ SemaglutideMutant

\section{HAEGTFTSDVSSYLEGQAAKEFIA WLRVGRG}

Genetically, the B27Arg-B28Val mutant of semaglutide is engineered in such a way that it has a similar amino acid sequence as the native semaglutide but has a Val-Arg (V-R) exchange (Figure 1) of valine (Val)-arginine (Arg) sequence at positions 27 and 28 in the B chain of PDB ID: 4ZGM [2], similar to the way insulin lispro is designed 


\section{literature HXEGTFTSDVSSYLEGQAAKEFIAWLVRGRG 31 mutant HAEGTFTSDVSSYLEGQAAKEFIAWLRVGRG 31 * *************************** $* * *$}

Figure 1: An alignment of the amino acid sequences of native (from literature $[2,11])$ semaglutide and the B27Arg-B28Val mutant (supplementary file sema.pdb) semaglutide peptide backbones. In this figure, the residue numberings are different for the two sequences. For instance, positions 27 and 28 (of native semaglutide) corresponds to positions 33 and 34 (from literature $[2,11]$ ).

$[43,44,45,46,47]$.

To build a homology structural model of the V-R exchange (Figure 1) mutant (B27Arg-B28Val) of semaglutide-GLP-1R, the two sequences above ( $\geq$ GLP-1R and $>$ SemaglutideMutant) were plugged into the SWISS-MODEL homology modeling $[48,49]$ server in search of an experimental structural model, which led to the heterodimeric complex structure (PDB ID: 4ZGM [2]) with 98.69\% sequence homology to the two sequences above ( $\geq$ GLP-1R and $\geq$ SemaglutideMutant $)$. After the homology structural modeling [48], the UCSF Chimera software [50] was employed to minimize the energy level of the homology structural model. The energy-minimized model (supplementary file sema.pdb) was subsequently subject to a comprehensive set of electrostatic interaction analysis as described previously in [42].

\section{Results}

In the complex structure of the native semaglutide peptide backbone in complex with

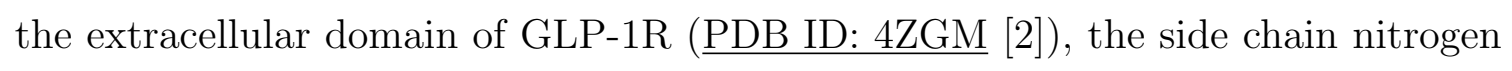


atoms of Arg28 of chain B are at least $12.4 \AA$ away from the side chain oxygen atoms of Glu104 of chain A, making interfacial salt bridges or hydrogen bonds practically impossible between the two interfacial residues. Furthermore, two sets of electrostatic interactions contribute towards the stabilization of structure (PDB ID: 4ZGM) of native semaglutide (chain B) bound to GLP-1R (chain A), including interfacial salt bridges between Lys20 of chain B (K20B) and Glu105 of chain A (E105A), and interfacial salt bridges and side chain hydrogen bond between Glu45 of chain A (E45A) and Arg30 of chain B (R30B), as listed in Tables 1 and 2 and illustrated in Figures 2 and 3 and 4.

\begin{tabular}{|l|c|c|c|c|c|}
\hline PDB file name & Residue A & Atom A & Residue B & Atom B & Distance $(\AA)$ \\
\hline sema.pdb & B_LYS_20 & NZ & A_GLU_105 & OE1 & 2.575 \\
\hline sema.pdb & B_LYS_20 & NZ & A_GLU_105 & OE2 & 2.713 \\
\hline sema.pdb & B_ARG_27 & NH2 & A_GLU_104 & OE2 & 3.694 \\
\hline sema.pdb & B_ARG_30 & NH1 & A_GLU_45 & OE1 & 3.734 \\
\hline sema.pdb & B_ARG_30 & NH1 & A_GLU_45 & OE2 & 3.775 \\
\hline
\end{tabular}

Table 1: Interfacial salt bridging network analysis of the homology structural model of the B27Arg-B28Val mutant of semaglutide peptide backbone in complex with the GLP-1 receptor extracellular domain (supplementary file sema.pdb). In this table, the residue naming scheme is Chain ID_residue name_residue number.

\begin{tabular}{|l|l|l|l|c|c|c|}
\hline PDB file name & Acceptor (A) & Donor (D) & Hydrogen $(\mathrm{H})$ & D-A $(\AA)$ & H-A $(\AA)$ & $\angle A D H\left(^{\circ}\right)$ \\
\hline sema.pdb & OE1, A_GLU_45 & NE, B_ARG_30 & HE, B_ARG_30 & 2.72 & 1.75 & 14.43 \\
\hline
\end{tabular}


Table 2: Interfacial side chain hydrogen bonding analysis of the homology structural model of the B27Arg-B28Val mutant of semaglutide peptide backbone in complex with the GLP-1 receptor extracellular domain (supplementary file sema.pdb). In this table, the residue naming scheme is Chain ID_residue name_residue number, $\angle A D H$ represents the angle formed by acceptor $(\mathrm{A})$, donor $(\mathrm{D})$ and hydrogen $(\mathrm{H})$ $(\angle A D H)$. 


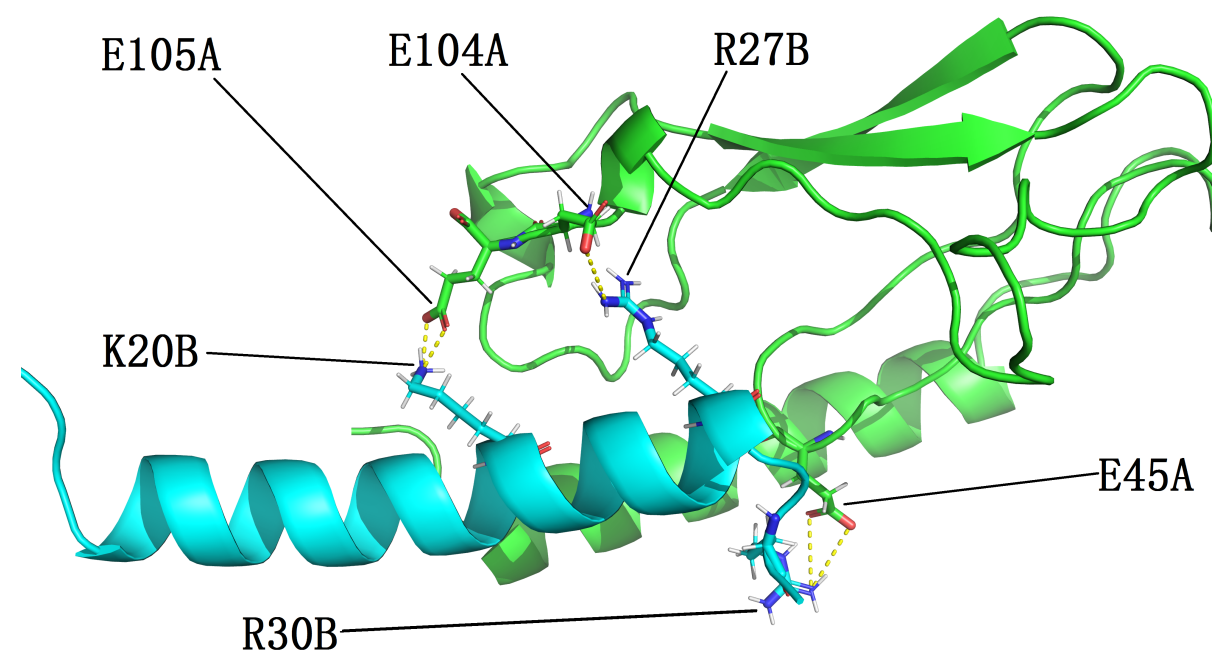

Figure 2: An overview of the homology structural model of the B27Arg-B28Val mutant of semaglutide peptide backbone (cyan cartoon) in complex with the GLP-1 receptor extracellular domain (green cartoon). In this figure, six amino acid residues are highlighted as sticks because they are involved in a set of interfacial structurestabilizing electrostatic interactions, including Lys20 of chain B (K20B), Glu105 of chain A (E105A), Glu104 of chain A (E104A), Arg27 of chain B (R27B), Glu45 of chain A (E45A) and Arg30 of chain B (R30B), all interfacial electrostatic interactions are indicated with yellow dotted lines. This figure is prepared by PyMol [51]. 


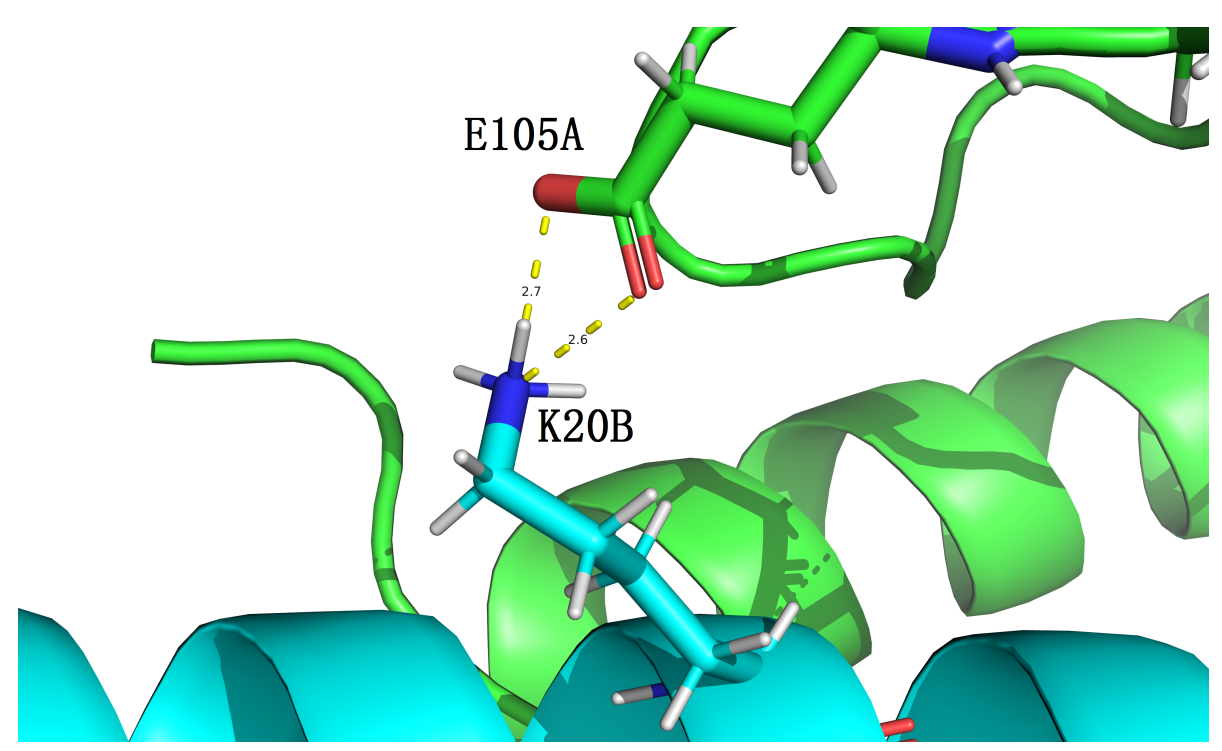

Figure 3: Homology structural model of the B27Arg-B28Val mutant of semaglutide peptide backbone (cyan cartoon) in complex with the GLP-1 receptor extracellular domain (green cartoon). In this figure, a set of interfacial salt bridges (yellow dotted lines) are highlighted between Lys20 of chain B (K20B) and Glu105 of chain A (E105A), with their details included in Table 1. This figure is prepared by PyMol $[51]$. 

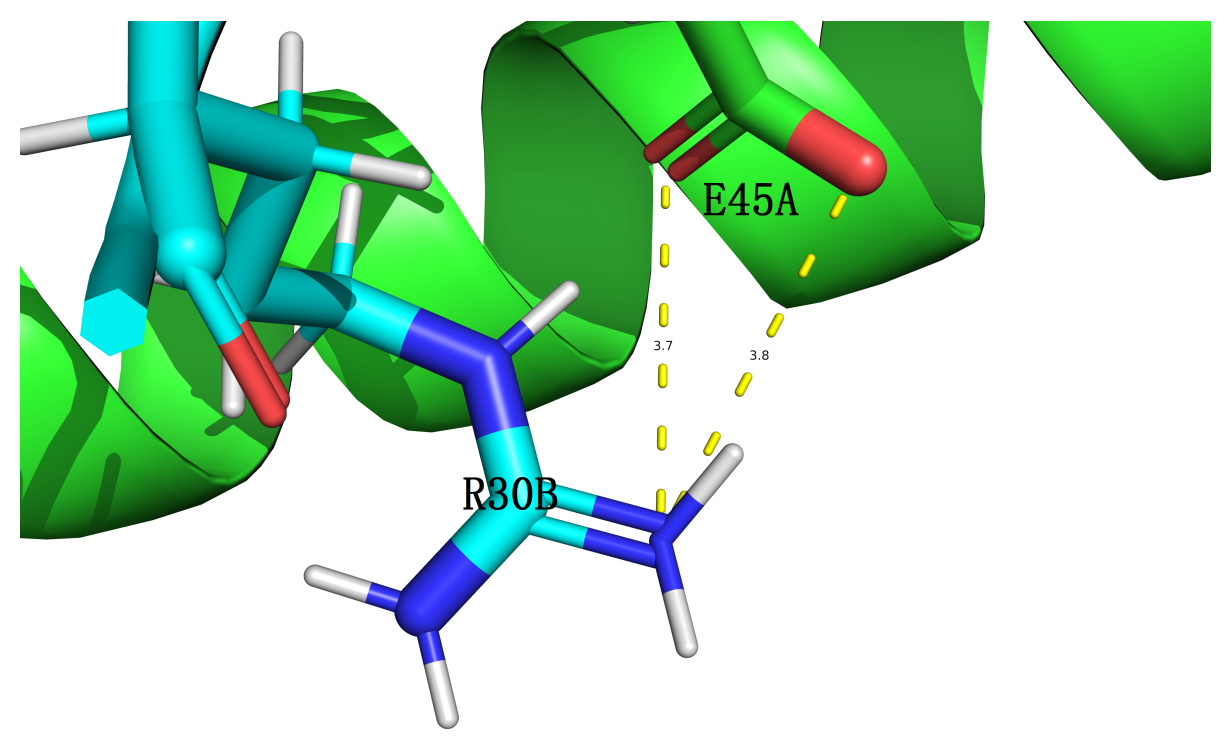

Figure 4: Homology structural model of the B27Arg-B28Val mutant of semaglutide peptide backbone (cyan cartoon) in complex with the GLP-1 receptor extracellular domain (green cartoon). In this figure, a set of interfacial salt bridges (yellow dotted lines) and one side chain hydrogen bond are highlighted between Glu45 of chain A (E45A) and Arg30 of chain B (R30B), with their details included in Tables 1 and 2. This figure is prepared by PyMol [51]. 
With respect to the homology structural model (supplementary file sema.pdb) of the V-R exchange (Figure 1) mutant (B27Arg-B28Val) of semaglutide in complex with GLP-1R, three sets of electrostatic interactions contribute towards the stabilization of structure (supplementary file sema.pdb) of V-R exchange mutant semaglutide (chain B) bound to GLP-1R (chain A), including interfacial salt bridges between Lys20 of chain B (K20B) and Glu105 of chain A (E105A), and between Glu104 of chain A (E104A) and Arg27 of chain B (R27B), and interfacial salt bridges and side chain hydrogen bond between Glu45 of chain A (E45A) and Arg30 of chain B (R30B), as listed in Tables 1 and 2 and illustrated in Figures 2, 3, 4 and 5.

Of further biophysical interest is that the structure-stabilizing interfacial salt bridges (Figures 5) between Glu104 of chain A (E104A) and Arg27 of chain B (R27B) is entirely due to the V-R exchange in the semaglutide peptide backbone, as shown in Figure 1, while the other two sets of interfacial electrostatic interactions remains unchanged (Figures 2 and 3 and 4).

In addition, of the homology structural model (supplementary file sema.pdb) of the V-R exchange (Figure 1) mutant (B27Arg-B28Val) of semaglutide in complex with GLP-1R, further structural analysis revealed that in addition to the interfacial salt bridges listed in Table 1, the oppositely charged side chains of the three residue pairs (Figure 2) are still quite close spatially to each other, with their inter-atomic distances close to the distance cut-off [42] for structural salt bridge screening (Table 3).

\begin{tabular}{|l|c|c|c|c|c|c|}
\hline PDB file name & Residue A & Atom A & Residue B & Atom B & Distance $(\AA)$ & Notes \\
\hline sema.pdb & B_LYS_20 & NZ & A_GLU_105 & OE1 & 2.575 & Yes \\
\hline sema.pdb & B_LYS_20 & NZ & A_GLU_105 & OE2 & 2.713 & Yes \\
\hline sema.pdb & B_ARG_27 & NH1 & A_GLU_104 & OE1 & 5.947 & No \\
\hline sema.pdb & B_ARG_27 & NH1 & A_GLU_104 & OE2 & 5.095 & No \\
\hline
\end{tabular}




\begin{tabular}{|l|c|c|c|c|c|c|}
\hline sema.pdb & B_ARG_27 & NH2 & A_GLU_104 & OE1 & 5.246 & no \\
\hline sema.pdb & B_ARG_27 & NH2 & A_GLU_104 & OE2 & 3.694 & Yes \\
\hline sema.pdb & B_ARG_30 & NH1 & A_GLU_45 & OE1 & 3.734 & Yes \\
\hline sema.pdb & B_ARG_30 & NH1 & A_GLU_45 & OE2 & 3.775 & Yes \\
\hline sema.pdb & B_ARG_30 & NH2 & A_GLU_45 & OE1 & 4.838 & No \\
\hline sema.pdb & B_ARG_30 & NH2 & A_GLU_45 & OE2 & 5.577 & No \\
\hline
\end{tabular}

Table 3: Interfacial salt bridging network analysis of the homology structural model of the B27Arg-B28Val mutant of semaglutide peptide backbone in complex with the GLP-1 receptor extracellular domain (supplementary file sema.pdb). In this table, the residue naming scheme is Chain ID_residue name_residue number. In the last column of this table, Yes means that a normal salt bridge is formed because the inter-atomic distance is shorter than $4 \AA$, while No means that no normal salt bridge is formed because the inter-atomic distance is longer than $4 \AA$, the distance cut-off for structural salt bridge screening used in [42].

\section{Conclusion}

For the first time, this short article puts forward a homology structural model (supplementary file sema.pdb) of the B27Arg-B28Val mutant of semaglutide peptide backbone in complex with the extracellular domain of GLP-1R. With an in-depth structural analysis of it, this short article argues that biophysically, this B27ArgB28Val mutant of semaglutide is able to bind GLP-1R tighter than semaglutide itself, hoping that the R\&D of a novel semaglutide analogue with the V-R exchange (Figure 1) is within our reach in the foreseeable future $[52,53,54,55]$. 


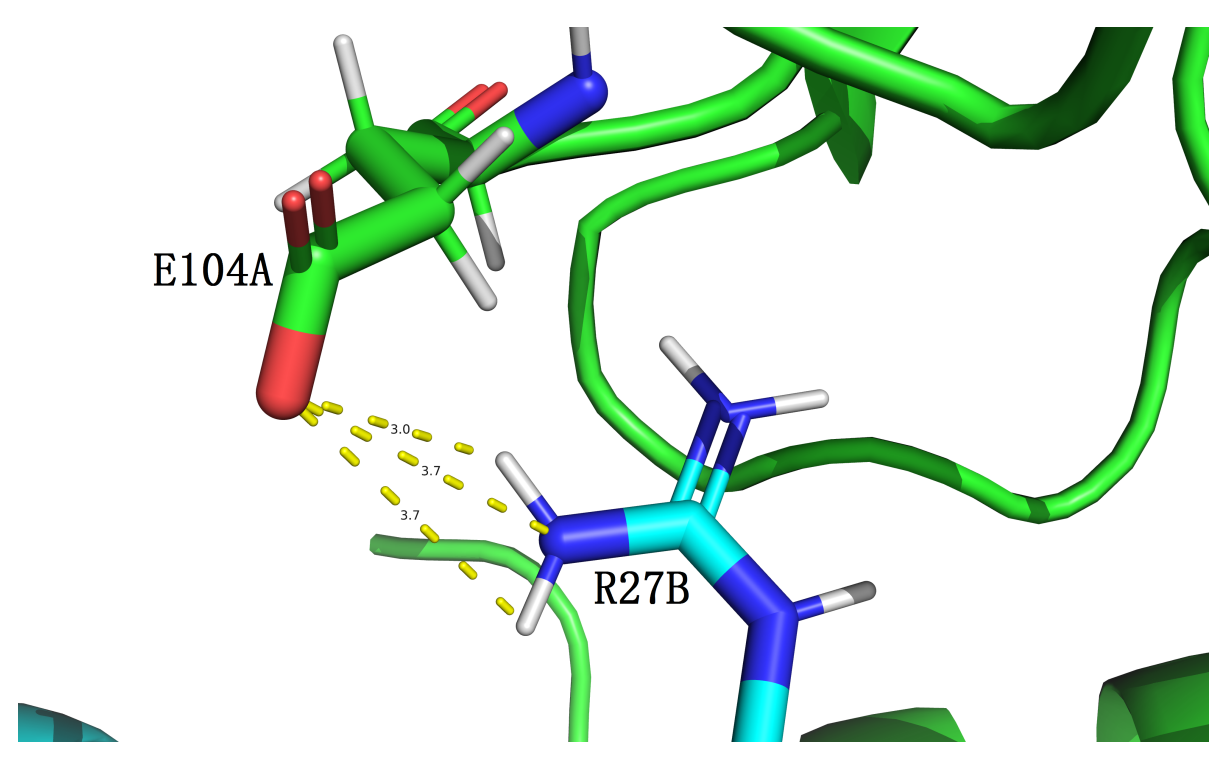

Figure 5: Homology structural model of the B27Arg-B28Val mutant of semaglutide peptide backbone (cyan cartoon) in complex with the GLP-1 receptor extracellular domain (green cartoon). In this figure, a set of interfacial salt bridges (yellow dotted lines) are highlighted between Glu104 of chain A (E104A) and Arg27 of chain B (R27B), with their details included in Table 1. This figure is prepared by PyMol [51]. 


\section{Conflict of interest}

None. 


\section{References}

[1] Ahrén B, Atkin SL, Charpentier G, Warren ML, Wilding JPH, Birch S, et al. Semaglutide induces weight loss in subjects with type 2 diabetes regardless of baseline BMI or gastrointestinal adverse events in the SUSTAIN 1 to 5 trials. Diabetes, Obesity and Metabolism. 2018 Jun;20(9):2210-2219.

[2] Lau J, Bloch P, Schäffer L, Pettersson I, Spetzler J, Kofoed J, et al. Discovery of the Once-Weekly Glucagon-Like Peptide-1 (GLP-1) Analogue Semaglutide. Journal of Medicinal Chemistry. 2015 Sep;58(18):7370-7380.

[3] Husain M, Birkenfeld AL, Donsmark M, Dungan K, Eliaschewitz FG, Franco DR, et al. Oral Semaglutide and Cardiovascular Outcomes in Patients with Type 2 Diabetes. New England Journal of Medicine. 2019 aug;381(9):841-851.

[4] Røder ME. Clinical potential of treatment with semaglutide in type 2 diabetes patients. Drugs in Context. 2019 dec;8:1-11.

[5] Aroda VR, Rosenstock J, Terauchi Y, Altuntas Y, Lalic NM, Villegas ECM, et al. PIONEER 1: Randomized Clinical Trial of the Efficacy and Safety of Oral Semaglutide Monotherapy in Comparison With Placebo in Patients With Type 2 Diabetes. Diabetes Care. 2019 jun;42(9):1724-1732.

[6] Anderson SL, Beutel TR, Trujillo JM. Oral semaglutide in type 2 diabetes. Journal of Diabetes and its Complications. 2020 apr;34(4):107520.

[7] Pieber TR, Bode B, Mertens A, Cho YM, Christiansen E, Hertz CL, et al. Efficacy and safety of oral semaglutide with flexible dose adjustment versus sitagliptin in type 2 diabetes (PIONEER 7): a multicentre, open-label, randomised, phase 3a trial. The Lancet Diabetes \& Endocrinology. 2019 jul;7(7):528-539. 
[8] Lin CH, Shao L, Zhang YM, Tu YJ, Zhang Y, Tomlinson B, et al. An evaluation of liraglutide including its efficacy and safety for the treatment of obesity. Expert Opinion on Pharmacotherapy. 2019 dec;21(3):275-285.

[9] Pratley R, Amod A, Hoff ST, Kadowaki T, Lingvay I, Nauck M, et al. Oral semaglutide versus subcutaneous liraglutide and placebo in type 2 diabetes (PIONEER 4): a randomised, double-blind, phase 3a trial. The Lancet. 2019 jul;394(10192):39-50.

[10] Goncalves E, Bell DS. Efficacy of semaglutide versus liraglutide in clinical practice. Diabetes \& Metabolism. 2019 oct;

[11] Knudsen LB, Lau J. The Discovery and Development of Liraglutide and Semaglutide. Frontiers in Endocrinology. 2019 Apr;10:1-32.

[12] Gæde P, Johansen P, Tikkanen CK, Pollock RF, Hunt B, Malkin SJP. Management of Patients with Type 2 Diabetes with Once-Weekly Semaglutide Versus Dulaglutide, Exenatide ER, Liraglutide and Lixisenatide: A Cost-Effectiveness Analysis in the Danish Setting. Diabetes Therapy. 2019 may;10(4):1297-1317.

[13] Bonora BM, Avogaro A, Fadini GP. Effects of exenatide long-acting release on cardiovascular events and mortality in patients with type 2 diabetes: a systematic review and meta-analysis of randomized controlled trials. Acta Diabetologica. 2019 apr;56(9):1051-1060.

[14] Xiao Y, Sun L. Semaglutide in weight management. The Lancet. 2019 oct;394(10205):1226.

[15] Mosenzon O, Blicher TM, Rosenlund S, Eriksson JW, Heller S, Hels OH, et al. Efficacy and safety of oral semaglutide in patients with type 2 diabetes and 
moderate renal impairment (PIONEER 5): a placebo-controlled, randomised, phase 3a trial. The Lancet Diabetes \& Endocrinology. 2019 jul;7(7):515-527.

[16] Lingvay I, Catarig AM, Frias JP, Kumar H, Lausvig NL, le Roux CW, et al. Efficacy and safety of once-weekly semaglutide versus daily canagliflozin as addon to metformin in patients with type 2 diabetes (SUSTAIN 8): a double-blind, phase 3b, randomised controlled trial. The Lancet Diabetes \& Endocrinology. 2019 nov;7(11):834-844.

[17] Yang PY, Zou H, Amso Z, Lee C, Huang D, Woods AK, et al. New Generation Oxyntomodulin Peptides with Improved Pharmacokinetic Profiles Exhibit Weight Reducing and Anti-Steatotic Properties in Mice. Bioconjugate Chemistry. 2020 apr;31(4):1167-1176.

[18] Han J, Fu J, Yang Q, Zhou F, Chen X, Li C, et al. Rational design and biological evaluation of gemfibrozil modified Xenopus GLP-1 derivatives as longacting hypoglycemic agents. European Journal of Medicinal Chemistry. 2020 jul;198:112389.

[19] Rodbard HW, Rosenstock J, Canani LH, Deerochanawong C, Gumprecht J, Lindberg SØ, et al. Oral Semaglutide Versus Empagliflozin in Patients With Type 2 Diabetes Uncontrolled on Metformin: The PIONEER 2 Trial. Diabetes Care. 2019 sep;42(12):2272-2281.

[20] Bækdal TA, Borregaard J, Hansen CW, Thomsen M, Anderson TW. Effect of Oral Semaglutide on the Pharmacokinetics of Lisinopril, Warfarin, Digoxin, and Metformin in Healthy Subjects. Clinical Pharmacokinetics. 2019 apr;58(9):11931203. 
[21] Zinman B, Aroda VR, Buse JB, Cariou B, Harris SB, Hoff ST, et al. Efficacy, Safety, and Tolerability of Oral Semaglutide Versus Placebo Added to Insulin With or Without Metformin in Patients With Type 2 Diabetes: The PIONEER 8 Trial. Diabetes Care. 2019 sep;42(12):2262-2271.

[22] Hunt B, Malkin SJP, Moes RGJ, Huisman EL, Vandebrouck T, Wolffenbuttel BHR. Once-weekly semaglutide for patients with type 2 diabetes: a costeffectiveness analysis in the Netherlands. BMJ Open Diabetes Research \& Care. 2019 oct;7(1):e000705.

[23] Nuhoho S, Gupta J, Hansen BB, Fletcher-Louis M, Dang-Tan T, Paine A. Orally Administered Semaglutide Versus GLP-1 RAs in Patients with Type 2 Diabetes Previously Receiving 1-2 Oral Antidiabetics: Systematic Review and Network Meta-Analysis. Diabetes Therapy. 2019 oct;10(6):2183-2199.

[24] Oral Semaglutide and Cardiovascular Outcomes in Type 2 Diabetes. New England Journal of Medicine. 2019 nov;381(21):2075-2077.

[25] Wilding JPH. Semaglutide in weight management - Author's reply. The Lancet. 2019 oct;394(10205):1226-1227.

[26] Bucheit JD, Pamulapati LG, Carter N, Malloy K, Dixon DL, Sisson EM. Oral Semaglutide: A Review of the First Oral Glucagon-Like Peptide 1 Receptor Agonist. Diabetes Technology \& Therapeutics. 2020 jan;22(1):10-18.

[27] Kanters S, Wilkinson L, Vrazic H, Sharma R, Lopes S, Popoff E, et al. Comparative efficacy of once-weekly semaglutide versus SGLT-2 inhibitors in patients inadequately controlled with one to two oral antidiabetic drugs: a systematic literature review and network meta-analysis. BMJ Open. 2019 jul;9(7):e023458. 
[28] Avgerinos I, Michailidis T, Liakos A, Karagiannis T, Matthews DR, Tsapas A, et al. Oral semaglutide for type 2 diabetes: A systematic review and metaanalysis. Diabetes, Obesity and Metabolism. 2019 nov;22(3):335-345.

[29] Newsome P, Francque S, Harrison S, Ratziu V, Gaal LV, Calanna S, et al. Effect of semaglutide on liver enzymes and markers of inflammation in subjects with type 2 diabetes and/or obesity. Alimentary Pharmacology \& Therapeutics. 2019 jun;50(2):193-203.

[30] Rasmussen MF. The development of oral semaglutide, an oral GLP-1 analog, for the treatment of type 2 diabetes. Diabetology International. 2020 jan;11(2):76-86.

[31] Abramson A, Halperin F, Kim J, Traverso G. Quantifying the Value of Orally Delivered Biologic Therapies: A Cost-Effectiveness Analysis of Oral Semaglutide. Journal of Pharmaceutical Sciences. 2019 sep;108(9):3138-3145.

[32] Thethi TK, Pratley R, Meier JJ. Efficacy, safety and cardiovascular outcomes of once-daily oral semaglutide in patients with type 2 diabetes: The PIONEER programme. Diabetes, Obesity and Metabolism. 2020 may;.

[33] Malkin SJP, Russel-Szymczyk M, Psota M, Hlavinkova L, Hunt B. The Management of Type 2 Diabetes with Once-Weekly Semaglutide Versus Dulaglutide: A Long-Term Cost-Effectiveness Analysis in Slovakia. Advances in Therapy. 2019 jun;36(8):2034-2051.

[34] Lee YS, Jun HS. Anti-diabetic actions of glucagon-like peptide-1 on pancreatic beta-cells. Metabolism. 2014 Jan;63(1):9-19. 
[35] Kim KK. Understanding the Mechanism of Action and Clinical Implications of Anti-Obesity Drugs Recently Approved in Korea. Korean Journal of Family Medicine. 2019 Mar;40(2):63-71.

[36] Hamal S, Cherukuri L, Shaikh K, Kinninger A, Doshi J, Birudaraju D, et al. Effect of semaglutide on coronary atherosclerosis progression in patients with type II diabetes. Coronary Artery Disease. 2020 may;31(3):306-314.

[37] Blundell TL. Protein crystallography and drug discovery: recollections of knowledge exchange between academia and industry. IUCrJ. 2017 Jun;4(4):308321.

[38] Aschenbrenner DS. New Drug for Type 2 Diabetes. AJN, American Journal of Nursing. 2020 jan;120(1):25.

[39] Cowart K. Oral Semaglutide: First-in-Class Oral GLP-1 Receptor Agonist for the Treatment of Type 2 Diabetes Mellitus. Annals of Pharmacotherapy. 2019 nov;54(5):478-485.

[40] Nadkarni P, Chepurny OG, Holz GG. Regulation of Glucose Homeostasis by GLP-1. In: Progress in Molecular Biology and Translational Science. Elsevier; 2014. p. 23-65.

[41] Bucheit JD, Pamulapati LG, Carter N, Malloy K, Dixon DL, Sisson EM. Oral Semaglutide: A Review of the First Oral Glucagon-Like Peptide 1 Receptor Agonist. Diabetes Technology \& Therapeutics. 2020 Jan;22(1):10-18.

[42] Li W. How do SMA-linked mutations of SMN1 lead to structural/functional deficiency of the SMA protein? PLOS ONE. 2017 jun;12(6):e0178519. 
[43] Kaiserman K, Jung H, Benabbad I, Karges B, Polak M, Rosilio M. 20 Years of insulin lispro in pediatric type 1 diabetes: a review of available evidence. Pediatric Diabetes. 2016 Jul;18(2):81-94.

[44] Baeshen NA, Baeshen MN, Sheikh A, Bora RS, Ahmed M, Ramadan H, et al. Cell factories for insulin production. Microbial Cell Factories. 2014 Oct;13(1).

[45] Walsh G, Murphy B, editors. Biopharmaceuticals, an Industrial Perspective. Springer Netherlands; 1999.

[46] Insulin Lispro. New England Journal of Medicine. 2003 Oct;349(15):1487-1487.

[47] Zaykov AN, Mayer JP, DiMarchi RD. Pursuit of a perfect insulin. Nature Reviews Drug Discovery. 2016 Mar;15(6):425-439.

[48] Waterhouse A, Bertoni M, Bienert S, Studer G, Tauriello G, Gumienny R, et al. SWISS-MODEL: homology modelling of protein structures and complexes. Nucleic Acids Research. 2018 May;46(W1):W296-W303.

[49] Biasini M, Bienert S, Waterhouse A, Arnold K, Studer G, Schmidt T, et al. SWISS-MODEL: modelling protein tertiary and quaternary structure using evolutionary information. Nucleic Acids Research. 2014 apr;42(W1):W252-W258.

[50] Pettersen EF, Goddard TD, Huang CC, Couch GS, Greenblatt DM, Meng EC, et al. UCSF Chimera: A visualization system for exploratory research and analysis. Journal of Computational Chemistry. 2004;25(13):1605-1612.

[51] DeLano WL. Pymol: An open-source molecular graphics tool. CCP4 Newsletter On Protein Crystallography. 2002;40:82-92. 
[52] Tsilingiris D, Liatis S, Dalamaga M, Kokkinos A. The Fight Against Obesity Escalates: New Drugs on the Horizon and Metabolic Implications. Current Obesity Reports. 2020 may;9(2):136-149.

[53] Leiter LA, Bain SC, Bhatt DL, Buse JB, Mazer CD, Pratley RE, et al. The effect of glucagon-like peptide-1 receptor agonists liraglutide and semaglutide on cardiovascular and renal outcomes across baseline blood pressure categories: Analysis of the LEADER and SUSTAIN 6 trials. Diabetes, Obesity and Metabolism. 2020 jun; :

[54] Atkinson MA, Powers AC. Research digest: pioneering an oral GLP-1 receptor agonist. The Lancet Diabetes \& Endocrinology. 2019 dec;7(12):897.

[55] Greenhill C. T2DM treatment trial results. Nature Reviews Endocrinology. 2019 jun;15(9):499-499. 